UDC 517.9

\title{
STABILITY ANALYSIS OF A PREDATOR-PREY MODEL WITH PIECEWISE CONSTANT ARGUMENT OF GENERALIZED TYPE USING LYAPUNOV FUNCTIONS
}

АНАЛІЗ СТІЙКОСТІ МОДЕЛІ ХИИАК-ЖЕРТВА»
ІЗ КУСКОВО-СТАЛИМ АРГУМЕНТОМ УЗАГАЛЬНЕНОГО ТИПУ
3 ВИКОРИСТАННЯМ ФУНКЦИИ ЛЯПУНОВА

D. Aruğaslan, A. Özer

Süleyman Demirel Univ.

32260, Isparta, Turkey

e-mail: duyguarugaslan@sdu.edu.tr, ozer.atilla@hotmail.com

\begin{abstract}
In this study, we consider a Lotka-Volterra type predator-prey model with piecewise constant arguments of generalized type and investigate the stability of the positive equilibrium point of the proposed model. Although the model includes piecewise constant delays, we do not use Lyapunov functionals. We establish the stability conditions using Lyapunov functions of the corresponding model of ordinary differential equations. In order to illustrate the validity of our results, we present an appropriate example and numerical simulations.

Розглянуто модель «хижак-жертва» типу Лотки-Вольтерра із кусково-сталими аргументами та вивчено стійкість додатного положення рівноваги розглядуваної моделі. Незважаючи на те, щзо модель мае кусково-сталі запізнення, функціонали Ляпунова не використовуються. Отримано умови стійкості з використанням функцій Ляпунова для відповідної моделі звичайних диференціальних рівнянь. Для ілюстрації отриманих результатів наведено відповідний приклад та числові розрахунки.
\end{abstract}

1. Introduction and preliminaries. Differential equations with piecewise constant argument have been intensively developed [5, 7, 12, 15-20] since they were initiated in [9-11]. In the last few decades, this class of differential equations has attracted considerable attention due to their wide range of applications in biology, control theory, neural networks etc. $[3,4,6,8,13$, $21,25]$. However, reduction to discrete equations and application of numerical methods have been the main instrument of investigation for differential equations with piecewise constant argument $[3,9-12]$. That being the case, initial value problems only with integer-valued initial moments can be taken into consideration and thus stability analysis can not be set out in full.

Akhmet [1,2] has generalized differential equations with piecewise constant arguments by taking arbitrary piecewise constant functions as arguments and used a new approach based on the construction of an equivalent integral equation. By means of this approach, stability problems can be considered by taking any real number as an initial moment. Afterwards, in [7], Akhmet et al. have developed the Lyapunov method for the following differential equation with piecewise constant arguments of generalized type:

$$
x^{\prime}(t)=f(t, x(t), x(\beta(t))),
$$

(c) D. Aruğaslan, A. Özer, 2013 
where $x \in B(h), B(h)=\left\{x \in \mathbb{R}^{n}:\|x\|<h\right\}, t \in \mathbb{R}^{+}, \beta(t)=\theta_{i}$ if $t \in\left[\theta_{i}, \theta_{i+1}\right), i \in \mathbb{N}$ and $\theta_{i}, i \in \mathbb{N}$, is a fixed real-valued sequence satisfying $0=\theta_{0}<\theta_{1}<\ldots<\theta_{i}<\ldots$ with $\theta_{i} \rightarrow \infty$ as $i \rightarrow \infty$. Here it is assumed that $\mathbb{N}$ and $\mathbb{R}^{+}$are, respectively, the sets of natural numbers and nonnegative real numbers; $\mathbb{R}^{n}, n \in \mathbb{N}$, is the $n$-dimensional real space and $\|$. is the Euclidean norm in $\mathbb{R}^{n}$. Despite the piecewise constant delay, they have established the stability conditions using Lyapunov functions only, that is, no functionals have been used. The authors have utilized the total stability concept [26] which has enabled to make a connection between the stability of equations with piecewise constant arguments and the corresponding ordinary differential equations. With these ideas and the assumptions including $f(t, 0,0)=0$ for all $t \geq 0$, they have investigated the stability of the zero solution of (1.1).

By a solution of equation (1.1) on $\mathbb{R}^{+}$we mean a continuous function $x(t)$ with the following properties: the derivative $x^{\prime}(t)$ exists everywhere with the possible exception of the points $\theta_{i}$, $i \in \mathbb{N}$, where one-sided derivatives exist; (1.1) is satisfied on each interval $\left[\theta_{i}, \theta_{i+1}\right), i \in \mathbb{N}[1,6$, 7].

In the present paper, we shall consider the Lotka-Volterra system, which is still one of the most famous models of predator-prey interactions in an ecosystem due to its theoretical and practical significances. Using the results of the paper [7], we aim to investigate the stability of Lotka - Volterra predator-prey model with piecewise constant arguments of generalized type.

It is well recognized that models of population dynamics under certain conditions do not satisfy realities. Naturally, more realistic and interesting models of populations should take the short term perturbations and time delays into account [14, 22, 23]. In [14], the authors studied the stability and Hopf bifurcation for the following delayed predator-prey system:

$$
\begin{aligned}
& N^{\prime}(t)=N(t)\left[r_{1}-a_{11} N(t-\tau)-a_{12} P(t)\right], \\
& P^{\prime}(t)=P(t)\left[-r_{2}+a_{21} N(t)-a_{22} P(t-\tau)\right],
\end{aligned}
$$

where $N(t)$ and $P(t)$ denote, respectively, the population densities of prey and predator at time $t ; r_{1}>0$ and $r_{2}>0$ are the intrinsic growth rate of the prey and the death rate of the predator, respectively; the parameters $a_{i j}, i, j=1,2$, are all positive constants. Assuming $a_{21} r_{1}>a_{11} r_{2}$, system (1.2) has a unique positive equilibrium $E=\left(N^{*}, P^{*}\right)$, where

$$
N^{*}=\frac{a_{22} r_{1}+a_{12} r_{2}}{a_{11} a_{22}+a_{12} a_{21}}
$$

and

$$
P^{*}=\frac{a_{21} r_{1}-a_{11} r_{2}}{a_{11} a_{22}+a_{12} a_{21}} .
$$

We aim to incorporate the piecewise constant argument of generalized type $\beta(t)$ defined in (1.1) into the model (1.2) and then investigate the stability of the positive equilibrium $E$. That is, we shall consider the model given by

$$
\begin{gathered}
N^{\prime}(t)=N(t)\left[r_{1}-a_{11} N(\beta(t))-a_{12} P(t)\right], \\
P^{\prime}(t)=P(t)\left[-r_{2}+a_{21} N(t)-a_{22} P(\beta(t))\right],
\end{gathered}
$$


where $N$ and $P$ lie in the circle centered at the point $E$ with radius $h$. We choose $h$ in a way that the circle stays in the first quadrant of the $N P$-plane.

Let us make the linear transformation $x_{1}=N-N^{*}$ and $x_{2}=P-P^{*}$. By this change of variables, the equilibrium point $E$ is mapped into the origin of the $x_{1} x_{2}$-plane. Then, system (1.3) can be written as follows:

$$
\begin{gathered}
x_{1}^{\prime}(t)=\left(x_{1}(t)+N^{*}\right)\left(-a_{11} x_{1}(\beta(t))-a_{12} x_{2}(t)\right), \\
x_{2}^{\prime}(t)=\left(x_{2}(t)+P^{*}\right)\left(a_{21} x_{1}(t)-a_{22} x_{2}(\beta(t))\right) .
\end{gathered}
$$

Let $x(t)=\left(x_{1}(t), x_{2}(t)\right)$ and $x(\beta(t))=\left(x_{1}(\beta(t)), x_{2}(\beta(t))\right)$. By defining

$$
\begin{gathered}
f_{1}(x(t), x(\beta(t))):=\left(x_{1}(t)+N^{*}\right)\left(-a_{11} x_{1}(\beta(t))-a_{12} x_{2}(t)\right), \\
f_{2}(x(t), x(\beta(t))):=\left(x_{2}(t)+P^{*}\right)\left(a_{21} x_{1}(t)-a_{22} x_{2}(\beta(t))\right),
\end{gathered}
$$

we can see that system (1.4) can be expressed as $x^{\prime}(t)=f(x(t), x(\beta(t)))$, where $x=\left(x_{1}, x_{2}\right)^{T}$ and $f=\left(f_{1}, f_{2}\right)^{T}$.

We can observe that system (1.4) satisfies the following conditions:

$\left(\mathrm{C}_{1}\right) f(u, v) \in C(B(h) \times B(h))$ is an $2 \times 1$ real-valued function;

$\left(\mathrm{C}_{2}\right) f(0,0)=0$

$\left(\mathrm{C}_{3}\right) f$ satisfies a Lipschitz condition with constants $\ell_{1}, \ell_{2}$, i.e.,

$$
\|f(x, y)-f(u, v)\| \leq \ell_{1}\|x-u\|+\ell_{2}\|y-v\|,
$$

for all $x, y, u, v \in B(h)$, where $\ell_{1}=\sqrt{2} \max \left\{\left(a_{12}+a_{11}+a_{21}\right) h+a_{21} P^{*},\left(a_{21}+a_{22}+a_{12}\right) h+\right.$ $\left.+a_{12} N^{*}\right\}$ and $\ell_{2}=\sqrt{2} \max \left\{a_{11}\left(N^{*}+h\right), a_{22}\left(P^{*}+h\right)\right\}$.

Let us prove the condition $\left(\mathrm{C}_{3}\right)$ :

$$
\begin{array}{rl}
\| f & f(x, y)-f(u, v) \|= \\
= & \left\|\begin{array}{c}
-a_{11} x_{1} y_{1}-a_{12} x_{1} x_{2}-a_{11} N^{*} y_{1}-a_{12} N^{*} x_{2}+a_{11} u_{1} v_{1}+a_{12} u_{1} u_{2}+a_{11} N^{*} v_{1}+a_{12} N^{*} u_{2} \\
a_{21} x_{1} x_{2}-a_{22} x_{2} y_{2}+a_{21} P^{*} x_{1}-a_{22} P^{*} y_{2}-a_{21} u_{1} u_{2}+a_{22} u_{2} v_{2}-a_{21} P^{*} u_{1}+a_{22} P^{*} v_{2}
\end{array}\right\|= \\
= & \left\|\begin{array}{c}
\left(-a_{12} x_{2}-a_{11} y_{1}\right)\left(x_{1}-u_{1}\right)+\left(-a_{11} N^{*}-a_{11} u_{1}\right)\left(y_{1}-v_{1}\right)+\left(-a_{12} N^{*}-a_{12} u_{1}\right)\left(x_{2}-u_{2}\right) \\
\left(a_{21} x_{1}-a_{22} y_{2}\right)\left(x_{2}-u_{2}\right)+\left(-a_{22} P^{*}-a_{22} u_{2}\right)\left(y_{2}-v_{2}\right)+\left(a_{21} P^{*}+a_{21} u_{2}\right)\left(x_{1}-u_{1}\right)
\end{array}\right\| \leq \\
\leq & \left(\left|-a_{12} x_{2}-a_{11} y_{1}\right|+\left|a_{21} P^{*}+a_{21} u_{2}\right|\right)\left|x_{1}-u_{1}\right|+\left(\left|a_{21} x_{1}-a_{22} y_{2}\right|+\mid-a_{12} N^{*}-\right. \\
& \left.-a_{12} u_{1} \mid\right)\left|x_{2}-u_{2}\right|+\left|-a_{11} N^{*}-a_{11} u_{1}\right|\left|y_{1}-v_{1}\right|+\left|-a_{22} P^{*}-a_{22} u_{2}\right|\left|y_{2}-v_{2}\right| \leq \\
\leq & \left(a_{12}\left|x_{2}\right|+a_{11}\left|y_{1}\right|+a_{21} P^{*} a_{21}\left|u_{2}\right|\right)\left|x_{1}-u_{1}\right|+\left(a_{21}\left|x_{1}\right|+a_{22}\left|y_{2}\right|+a_{12} N^{*}+a_{12}\left|u_{1}\right|\right)\left|x_{2}-u_{2}\right|+ \\
& +\left(a_{11} N^{*}+a_{11}\left|u_{1}\right|\right)\left|y_{1}-v_{1}\right|+\left(a_{22} P^{*}+a_{22}\left|u_{2}\right|\right)\left|y_{2}-v_{2}\right| \leq \\
\leq & \left(\left(a_{12}+a_{11}+a_{21}\right) h+a_{21} P^{*}\right)\left|x_{1}-u_{1}\right|+\left(\left(a_{21}+a_{22}+a_{12}\right) h+a_{12} N^{*}\right)\left|x_{2}-u_{2}\right|+ \\
& +\left(a_{11}\left(N^{*}+h\right)\right)\left|y_{1}-v_{1}\right|+\left(a_{22}\left(P^{*}+h\right)\right)\left|y_{2}-v_{2}\right| \leq \ell_{1}\|x-u\|+\ell_{2}\|y-v\|,
\end{array}
$$


which verifies (1.5). As a result, Lipschitz condition, that is, condition $\left(\mathrm{C}_{3}\right)$ is fulfilled for the function in the right-hand side of the model (1.4).

In what follows, we shall assume that the following conditions are satisfied:

$\left(\mathrm{C}_{4}\right)$ there exists a constant $\theta>0$ such that $\theta_{i+1}-\theta_{i} \leq \theta, i \in \mathbb{N}$;

$\left.\left(\mathrm{C}_{5}\right)\right] \theta\left[\ell_{2}+\ell_{1}\left(1+\ell_{2} \theta\right) e^{\ell_{1} \theta}\right]<1$

$\left(\mathrm{C}_{6}\right) \theta\left(\ell_{1}+2 \ell_{2}\right) e^{\ell_{1} \theta}<1$.

2. Main results. We give now some definitions and preliminary results which enable us to investigate stability of the zero solution $x=0$ of (1.4).

Definition 2.1 [2]. The zero solution of (1.4) is said to be,

(i) stable if for any $\varepsilon>0$ and $t_{0} \in \mathbb{R}^{+}$, there exists $a \delta=\delta\left(t_{0}, \varepsilon\right)>0$ such that $\left\|x_{0}\right\|<\delta$ implies $\left\|x\left(t, t_{0}, x_{0}\right)\right\|<\varepsilon$ for all $t \geq t_{0}$

(ii) uniformly stable if $\delta$ is independent of $t_{0}$.

Definition 2.2 [2]. The zero solution of (1.4) is said to be uniformly asymptotically stable if it is uniformly stable and there is a $\delta_{0}>0$ such that for every $\varepsilon>0$ and $t_{0} \in \mathbb{R}^{+}$, there exists a $T=T(\varepsilon)>0$ such that $\left\|x\left(t, t_{0}, x_{0}\right)\right\|<\varepsilon$ for all $t>t_{0}+T$ whenever $\left\|x_{0}\right\|<\delta_{0}$.

The following lemma plays a crucial role in the proof of the stability theorem.

Lemma 2.1 [7]. If the conditions $\left(C_{4}\right),\left(C_{5}\right)$ are fulfilled, then for a solution $x(t)$ of (1.4) we have the estimation

$$
\|x(\beta(t))\| \leq m\|x(t)\|
$$

for all $t \in \mathbb{R}^{+}$, where $m=\left\{1-\theta\left[\ell_{2}+\ell_{1}\left(1+\ell_{2} \theta\right) e^{\ell_{1} \theta}\right]\right\}^{-1}$.

Next, we need the following theorem which provides conditions for the existence and uniqueness of solutions on $\mathbb{R}^{+}$. Since the proof of the assertion is almost identical to the one given in [1], we omit it here.

Theorem 2.1. Suppose that conditions $\left(C_{4}\right)-\left(C_{6}\right)$ are fulfilled. Then for every $\left(t_{0}, x_{0}\right) \in \mathbb{R}^{+} \times$ $\times B(h)$ there exists a unique solution $x(t)=x\left(t, t_{0}, x_{0}\right)$ of $(1.4)$ on $\mathbb{R}^{+}$with $x\left(t_{0}\right)=x_{0}$.

In consideration of the paper [7] we shall make use of the following system of ordinary differential equations that corresponds to (1.4), that is the system given by

$$
\begin{gathered}
x_{1}^{\prime}(t)=\left(x_{1}(t)+N^{*}\right)\left(-a_{11} x_{1}(t)-a_{12} x_{2}(t)\right):=g_{1}(x), \\
x_{2}^{\prime}(t)=\left(x_{2}(t)+P^{*}\right)\left(a_{21} x_{1}(t)-a_{22} x_{2}(t)\right):=g_{2}(x) .
\end{gathered}
$$

Let the time derivative of a function $V(x)$ with respect to system (2.1) be defined as

$$
V_{(3)}^{\prime}(x)=\frac{\partial V(x)}{\partial x} g(x)
$$

for all $x \in B(h)$.

Consider the function $F\left(x_{1}, x_{2}\right)=\sqrt{\frac{1}{\left(N^{*}+x_{1}\right)^{2}}+\frac{1}{\left(P^{*}+x_{2}\right)^{2}}}$ on the region $B(h)$. Since $F$ is a continuous function on a closed region it has a maximum value, say, $M$. Next, the following results follows. 
Theorem 2.2. Let the conditions $\left(C_{4}\right)-\left(C_{6}\right)$ be fulfilled. The zero solution of (1.4) is uniformly asymptotically stable if there exists a positive constant $\tau$ such that

$$
M \leq \frac{\min \left\{a_{11}-a_{21}, a_{22}-a_{12}\right\}-\tau}{\ell_{2}(1+m)}
$$

Proof. Let $x(t)=\left(x_{1}(t), x_{2}(t)\right.$ be a solution of (1.4) with $\left(x_{1}\left(t_{0}\right), x_{2}\left(t_{0}\right)\right) \in B(h)$. Consider a Lyapunov function defined for $t \geq t_{0}$ by

$$
V(x)=\left|\ln \left(x_{1}+N^{*}\right)-\ln N^{*}\right|+\left|\ln \left(x_{2}+P^{*}\right)-\ln P^{*}\right| .
$$

It is clear that $V$ is a positive definite and decreasing function. Hence, there exist strictly increasing functions $u, v \in C\left(\mathbb{R}^{+}, \mathbb{R}^{+}\right), u(0)=v(0)=0$ such that [24]

$$
u(\|x\|) \leq V(x) \leq v(\|x\|) \text { for all } \quad x \in B(h) .
$$

Let $h(x(t), x(\beta(t))):=f(x(t), x(\beta(t)))-f(x(t), x(t))=f(x(t), x(\beta(t)))-g(x(t))$.

If we evaluate the time derivative of $V$ along the solutions of (1.4), we find for $t \neq \theta_{i}$ that

$$
\begin{aligned}
V_{(1.4)}^{\prime}( & \left.x_{1}, x_{2}\right)=V_{(2.1)}^{\prime}\left(x_{1}, x_{2}\right)+\left\langle\frac{\partial V(x)}{\partial x}, h(x(t), x(\beta(t)))\right\rangle \leq \\
\leq & \operatorname{sgn}\left(\ln \left(x_{1}+N^{*}\right)-\ln N^{*}\right)\left(-a_{11} x_{1}-a_{12} x_{2}\right)+ \\
& +\operatorname{sgn}\left(\ln \left(x_{2}+P^{*}\right)-\ln P^{*}\right)\left(a_{21} x_{1}-a_{22} x_{2}\right)+\ell_{2}(1+m)\left\|\frac{\partial V(x)}{\partial x}\right\|\|x\| \leq \\
\leq & \operatorname{sgn}\left(x_{1}\right)\left(-a_{11} x_{1}-a_{12} x_{2}\right)+\operatorname{sgn}\left(x_{2}\right)\left(a_{21} x_{1}-a_{22} x_{2}\right)+\left\|\frac{\partial V(x)}{\partial x}\right\| \ell_{2}(1+m)\|x\| \leq \\
\leq & -a_{11}\left|x_{1}\right|-a_{12} \operatorname{sgn}\left(x_{1}\right) x_{2}+a_{21} \operatorname{sgn}\left(x_{2}\right) x_{1}-a_{22}\left|x_{2}\right|+\ell_{2}(1+m)\left\|\frac{\partial V(x)}{\partial x}\right\|\|x\| \leq \\
\leq & -a_{11}\left|x_{1}\right|+a_{12}\left|x_{2}\right|+a_{21}\left|x_{1}\right|-a_{22}\left|x_{2}\right|+\left\|\frac{\partial V(x)}{\partial x}\right\| \ell_{2}(1+m)\|x\|= \\
= & \left(a_{21}-a_{11}\right)\left|x_{1}\right|+\left(a_{12}-a_{22}\right)\left|x_{2}\right|+\ell_{2}(1+m)\left\|\frac{\partial V(x)}{\partial x}\right\|\|x\| \leq \\
\leq & {\left[\max \left\{a_{21}-a_{11}, a_{12}-a_{22}\right\}+\ell_{2}(1+m) F\left(x_{1}, x_{2}\right)\right]\|x\| \leq } \\
\leq & {\left[\max \left\{a_{21}-a_{11}, a_{12}-a_{22}\right\}+\ell_{2}(1+m) M\right]\|x\| \leq-\tau\|x\| . }
\end{aligned}
$$

Hence by Theorem 3.7 of [7], we conclude that the zero solution of (1.4) is uniformly asymptotically stable.

Theorem 2.2 is proved.

Since we consider a linear transformation of the axes, we can derive the next assertion. 
Theorem 2.3. Let the conditions $\left(C_{4}\right)-\left(C_{6}\right)$ be fulfilled. The equilibrium $E=\left(N^{*}, P^{*}\right)$ of (1.3) is uniformly asymptotically stable if there exists a positive constant $\tau$ such that

$$
M \leq \frac{\min \left\{a_{11}-a_{21}, a_{22}-a_{12}\right\}-\tau}{\ell_{2}(1+m)}
$$

3. Numerical simulations. As an example, we consider the system (1.3) with $r_{1}=500$, $r_{2}=1, a_{11}=40, a_{12}=1, a_{21}=30, a_{22}=20, \theta_{i}=i / 7000, i \in \mathbb{N}$, and $h=11.9$, i.e.,

$$
\begin{aligned}
& N^{\prime}(t)=N(t)[500-40 N(\beta(t))-P(t)], \\
& P^{\prime}(t)=P(t)[-1+30 N(t)-20 P(\beta(t))],
\end{aligned}
$$

which has the positive equilibrium point

$$
E=\left(N^{*}, P^{*}\right)=\left(\frac{10001}{830}, \frac{1496}{83}\right) \cong(12.05,18.02)
$$

It is clear that the system given by (3.1) satisfies the conditions $\left(\mathrm{C}_{1}\right)-\left(\mathrm{C}_{3}\right)$ with $\ell_{1}=1959.566686$ and $\ell_{2}=1354.782515$. We note that the model parameters are chosen in a way to satisfy conditions $\left(\mathrm{C}_{4}\right)-\left(\mathrm{C}_{6}\right)$.

By simple calculation, we find that $m=2.744189738$. Since $\theta=1 / 7000$, we see that

$$
\theta\left[\ell_{2}+\ell_{1}\left(1+\ell_{2} \theta\right) e^{\ell_{1} \theta}\right]=0.6355937106<1
$$

and

$$
\theta\left(\ell_{1}+2 \ell_{2}\right) e^{\ell_{1} \theta}=0.8824978508<1 .
$$

Hence, conditions $\left(\mathrm{C}_{4}\right)-\left(\mathrm{C}_{6}\right)$ are fullfilled. Taking

$$
V(x)=\left|\ln \left(x_{1}(t)+N^{*}\right)-\ln N^{*}\right|+\left|\ln \left(x_{2}(t)+P^{*}\right)-\ln P^{*}\right|
$$

as in the proof of Theorem 3, we find $\left\|\frac{\partial V(x)}{\partial x}\right\| \leq M=0.0007864841932$ for the system (3.1), and thus

$$
M \ell_{2}(1+m)-\min \left\{a_{11}-a_{21}, a_{22}-a_{12}\right\}=-6.010509547,
$$

which shows that all hypotheses of Theorem 2.3 are fulfilled. In order to see the validity of the theoretical results guaranteed by Theorem 2.3, we present below the solution graphs of prey $N$ and predator $P$ with respect to time $t$, respectively. For $t \in\left[\theta_{i}, \theta_{i+1}\right), i \in \mathbb{N}$, system (3.1) reduces to an ordinary differential equation. Hence, we can solve the system (3.1) numerically in each interval $\left[\theta_{i}, \theta_{i+1}\right), i \in \mathbb{N}$, by using MATLAB's built-in solver ode45. Consequently, we obtain that the positive equilibrium point $E$ of (3.1) is uniformly asymptotically stable as shown in Figures 1 and 2, which confirm the results of the Theorem 2.3. 


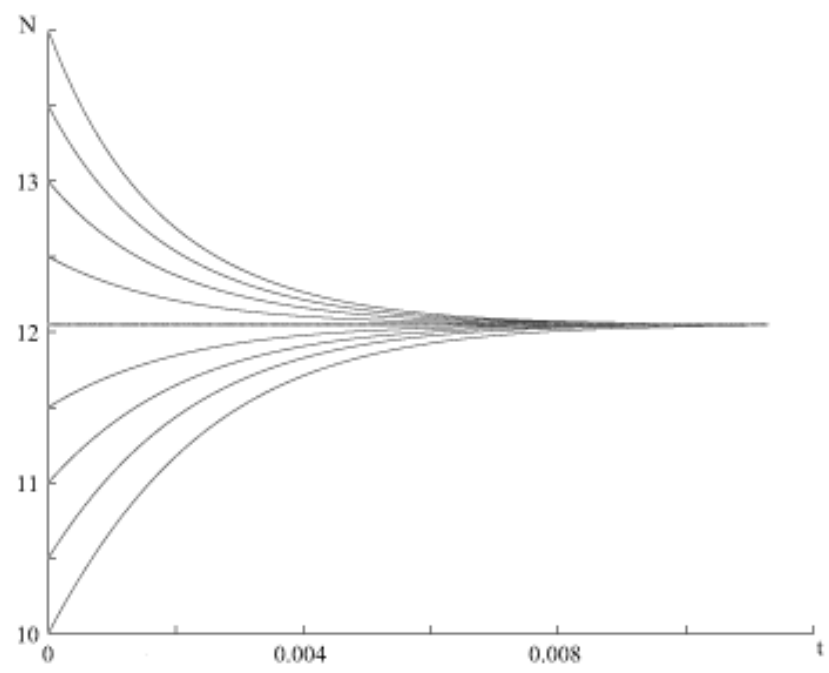

Fig. 1. Time response of the prey.

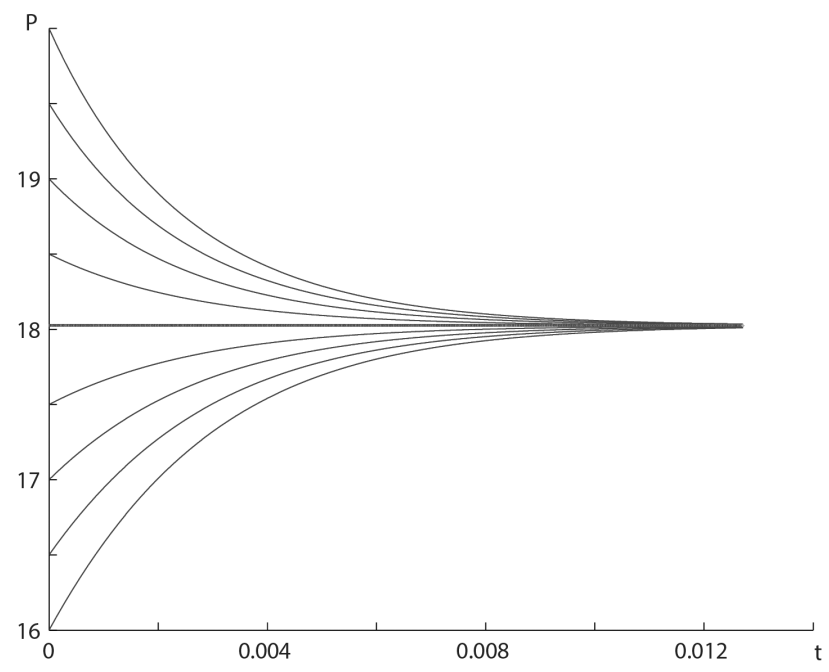

Fig. 2. Time response of the predator.

1. Akhmet M. U. Integral manifolds of differential equations with piecewise constant argument of generalized type // Nonlinear Anal. - 2007. - 66. - P. 367-383.

2. Akhmet M.U. Stability of differential equations with piecewise constant arguments of generalized type // Nonlinear Anal. - 2008. - 68. - P. 794-803.

3. Akhmet M. U., Öktem H., Pickl S. W., Weber G.-W. An anticipatory extension of Malthusian model // CASYS'05Seventh Int. Conf., AIP Conf. Proc. - 2006. - 839. - P. 260-264.

4. Akhmet M. U., Aruğaslan D., Liu X. Permanence of nonautonomous ratio-dependent predator-prey systems with piecewise constant argument of generalized type // Dynam. Contin. Discrete and Impulsive Syst. Ser. A. Math. Anal. - 2008. - 15, № 1. - P. 37-51.

5. Akhmet M. U., Aruğaslan D. Lyapunov-Razumikhin method for differential equations with piecewise constant argument // Discrete and Contin. Dynam. Syst. Ser. A. - 2009. - 25, № 2. - P. 457-466. 
6. Akhmet M. U., Aruğaslan D., Yllmaz E. Stability in cellular neural networks with a piecewise constant argument // J. Comput. and Appl. Math. - 2010. - 233, № 9. - P. 2365-2373.

7. Akhmet M. U., Aruğaslan D., Yılmaz E. Method of Lyapunov functions for differential equations with piecewise constant delay // J. Comput. and Appl. Math. - 2011. - 235, № 16. - P. 4554-4560.

8. Akhmet M. U., Aruğaslan D., Yllmaz E. Stability analysis of recurrent neural networks with piecewise constant argument of generalized type // Neural Networks. - 2010. - 23, № 7. - P. 805-811.

9. Busenberg S., Cooke K. L. Models of vertically transmitted diseases // Nonlinear Phenomena in Math. Sci. / Ed. V. Lakshmikantham. - New York: Acad. Press, 1982. - P. 179-187.

10. Wiener J., Cooke K. L. Retarded differential equations with piecewise constant delays // J. Math. Anal. and Appl. - 1984. - 99. - P. 265-297.

11. Wiener J., Shah S. M. Advanced differential equations with piecewise constant argument deviations // Int. J. Math. and Math. Sci. - 1983. - 6. - P. 671-703.

12. Wiener J. Generalized solutions of functional differential equations. - Singapore: World Sci., 1993.

13. Wiener J., Lakshmikantham V. A damped oscillator with piecewise constant time delay // Nonlinear Stud. 2000. - 7. - P. $78-84$.

14. Yan X. P., Li W. T. Hopf bifurcation and global periodic solutions in a delayed predator-prey system // Appl. Math. and Comput. - 2006. - 177, № 1. - P. 427-445.

15. Papaschinopoulos $G$. On the integral manifold for a system of differential equations with piecewise constant argument // J. Math. Anal. and Appl. - 1996. - 201. - P. 75 -90.

16. Wiener J., Cooke K. L. Oscillations in systems of differential equations with piecewise constant argument // J. Math. Anal. and Appl. - 1989. - 137. - P. 221-239.

17. Aftabizadeh A. R., Wiener J., Xu J. M. Oscillatory and periodic solutions of delay differential equations with piecewise constant argument // Proc. Amer. Math. Soc. - 1987. - 99. - P. 673-679.

18. Seifert $G$. Almost periodic solutions of certain differential equations with piecewise constant delays and almost periodic time dependence // J. Different. Equat. - 2000. - 164. - P. 451 - 458.

19. Wang G. Periodic solutions of a neutral differential equation with piecewise constant arguments // J. Math. Anal. and Appl. - 2007. - 326. - P. 736-747.

20. Cooke K. L., Wiener J. A survey of differential equation with piecewise continuous argument // Lect. Notes Math. - 1991. - 1475. - P. 1-15.

21. Muroya $Y$. Persistence, contractivity and global stability in logistic equations with piecewise constant delays // J. Math. Anal. and Appl. - 2002. - 270. - P. 602-635.

22. Kuang Y. Delay differential equations with applications in population dynamics. - New York: Acad. Press, 1993.

23. Liu X., Rohlf K. Impulsive control of a Lotka-Volterra system // IMA J. Math. Control Inform. - 1998. 15. - P. 269-284.

24. Miller R. K., Michel A. Ordinary differential equations. - New York: Acad. Press, 1982.

25. Dai L., Singh M. C. On oscillatory motion of spring-mass systems subjected to piecewise constant forces // J. Sound and Vibr. - 1994. - 173. - P. 217-232.

26. Hahn W. Stability of motion. - Berlin: Springer-Verlag, 1967.

Received 25.04.13,

after revision -10.07 .13$ 